\title{
Finite Element Analysis and Manufacture of Porous Structure Based on SLM Technique
}

\author{
Yaling Wang, Xianshuai Chen, Yuanxin Luo, Chunyu Zhang, Peng Zhang, and Wei Feng
}

\begin{abstract}
Selective laser melting (SLM) technique has been widely used in stomatology in recent years because of the advantages of its digitization and efficient. However, information about it is limited. What is more, studies have shown that porous structural dental implants based on SLM technique is beneficial to combination of implant and bone tissue. In this study, designing and manufacturing new types of porous implants based on SLM technique and performing simulation from the perspective of biomechanics are the main content. The results show that hollow structure has higher stress and strain in stress-concentration areas compared with solid structure. However, these differences would not form great influence. There are not obvious defects for the samples based on SLM technique on surface.
\end{abstract}

\section{Index Terms-Porous, FEA, manufacture, SLM.}

\section{INTRODUCTION}

The ability of SLM technique to produce components with very complex designs is one of the unique advantages of the technique that can be applied in biomedical applications. SLM technique melts metal powder to form the metal parts with complex structure based on the principle of superposition of layered manufacturing. Compared with other conventional manufacturing technology in this aspect, SLM technique has the irreplaceable advantages and become a hot research topic, as the academic dissertation [1]. Therefore, the technique is widely applied to many branches of stomatology, including oral and maxillofacial surgery, dental prosthesis, dental implantation and orthodontics, as said in the journal paper [2]. Nevertheless, the SLM study is still in its infancy, the mechanical properties, biocompatibility and materials are subject to further discussion. An understanding of the effect of implant design on its mechanical performance is required for any further improvement. Some studies have been performed to investigate the influence of a dental implant design on its mechanical properties, as described in the journal [3]. Study reveals that the different design is an important parameter needs to be considered and porous structure could enhance bone ingrowth. Therefore we investigate the effect of two different structures on mechanical properties based on SLM technique, which allows

Manuscript received April 22, 2016; revised July 19, 2016.

Yaling Wang and Yuanxin Luo are with the Chongqing University, CO 400044 China (e-mail: yl.wang@gzjanus.com)

Xianshuai Chen and Wei Feng are with the Shenzhen Institutes of Advanced Technology, Shenzhen, China, CO 518055 China (e-mail: xs.chen@giat.ac.cn).

Xianshuai Chen and Chunyu Zhang are with Foshan Angels Biotechnology Co., Ltd., Foshan, China, CO 528251 China.

Peng Zhang is with the Foshan Stomatology Hospital, Foshan, China. the separate control of pore structure, to determine the optimal porous structure for metal implants manufactured by this process. What's more, recent studies have shown that appropriate inner-porous structure promote the osseointegration of implant and bone tissue. Designing surface or entire porous structure dental implant can get better biomechanical performance. Due to the introduction of pore, which has become the research focus, the elastic modulus of porous titanium has effectively reduced the phenomenon of "stress shielding" has been avoided, and its unique porous structure is beneficial to the bone ingrowth. In this study, two different structure are simulated to determine if the porous structures could meet the clinical demands in mechanical properties when the ability of osseointegration is promoted.

\section{MATERIALS AND METHODS}

The first step of the numerical analysis is to draw three-dimensional geometric models of the jaw bone and the corresponding implant. In this study, two different structural implants are modeled, with the same dimensions of $4.8-\mathrm{mm}$ diameter, 7.6- $\mathrm{mm}$ length, $1.2-\mathrm{mm}$ pitch and $0.4-\mathrm{mm}$ thread depth in implant body. The implant system is modeled as an integrated structure. In this study, a straight abutment is designed with the maximum diameter of $4.6 \mathrm{~mm}$ and the length of $5.6 \mathrm{~mm}$. Cortical and cancellous bones are also modeled representing the cross-section of the posterior human mandible. Two-millimeter thickness of cortical bone was modeled around the cancellous bone and implant neck, a journal paper [4]. In order to understand the effect of porous structure on the distribution of stress and strain, a solid structure is modeled with $0.6-\mathrm{mm}$ pore in the surface of the implant. The other hollow structure is not only with $0.6-\mathrm{mm}$ pore in the surface but also has a hollow structure inside the implant body, as is shown in Fig. 1.
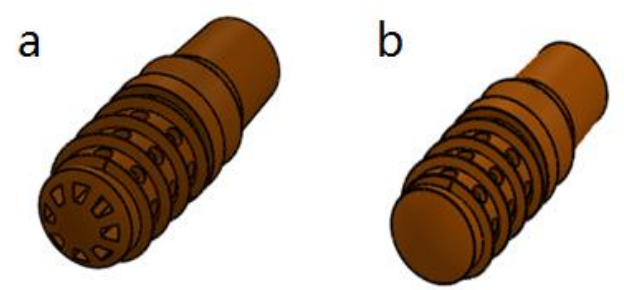

Fig. 1. Models of porous structure.

TABLE I: MECHANICAL PROPERTIES OF MATERIALS

\begin{tabular}{ccc}
\hline \hline Materials & Elastic modulus [GPa] & Poisson's ratio \\
\hline TA2 & 108 & 0.33 \\
Ceramic & 68.9 & 0.28 \\
Cortical bone & 13.7 & 0.3 \\
Cancellous bone & 1.37 & 0.3 \\
\hline \hline
\end{tabular}


A large number of studies have demonstrated when doing small deformation and static analysis, materials used in study were assumed to be continuous, homogeneous and isotropic. All of the materials applied to the implant systems are listed in tableI, just as journal paper [5]-[8]. The $100 \mathrm{~N}$ of the buccal oblique force is applied with $30^{\circ}$ to the long axis of the implant on the buccal cusp. The mesial and distal surfaces of alveolar bone were constrained in $\mathrm{x}, \mathrm{y}$ and $\mathrm{z}$ directions (displacements $=0$ ) as the boundary condition. For the simulation of osseointegration after implant placement, a bonded condition was set at the implant-bone interface and the simplified crowns were bonded with abutment.

The samples based on SLM technique are different from those based on CNC technique. Mechanical properties of TA2 have been greatly promoted, such as elongation,from the average of $17 \%$ to $27 \%$. At the same time, porosity would cause influence on the mechanical properties of formed parts. Some works has been performed to determine the relationship between the elastic modulus of porous parts and their relative porosity according to the (1):

$$
E^{*}=E \times(1-\Phi)^{2}
$$

where $E$ and $E^{*}$ are the elastic of materials and relative elastic of porous structure, $\Phi$ is the porosity. In this study, relative elastic of hollow structure with porosity of $19.6 \%$ could be calculated as $69.8 \mathrm{GPa}$ according to Table I and (1).

\section{RESULTS}

The 3D models established in solidworks 14.5 surface could be immediately generated in ANSYS Workbench Interface without translating into other intermediate format by seamless link between the two softwares. The distribution of stress and strain in the two models is mainly used to evaluate their mechanical properties.

Pure titanium parts manufactured by SLM technique are equipped with good mechanical property and biocompatibility, Tensile strength and yield strength are corresponding to $383 \mathrm{GPa}$ and $475 \mathrm{MPa}$ as journal paper [9].

The developed finite element models provide the magnitudes and distributions of the stress and strain throughout implant system. Fig. 2(a), (b) shows the stress distribution for the implant system of different structures. Stress distribution is almost the same for two different structures but the highest stress occurred for hollow structure, of around $140 \mathrm{MPa}$ at the crown-implant contact region. As for the implant, the maximum stress is located in the neck of implant, with the values of $134 \mathrm{MPa}$ in hollow structure and $130 \mathrm{MPa}$ in solid structure, $28.2 \%$ and $27.4 \%$ of yield strength of pure titanium parts based on SLM technique. The porous structures are the areas with high stress around the first lap of screw. Lower-middle parts of implants are not subjected to force basically. In the alveolar bone, the maximum stress is observed in crestal region around the socket in cortical bone, which corresponded with the clinical finding of maxillary crestal bone loss, as said in journal paper [10], with the values of $59 \mathrm{MPa}$ and $49 \mathrm{MPa}$. The stress of cancellous bone is so small that we can ignore its influence on implant system.

The distribution of strain in the implant system is consistent with that of stress. As can be seen from the pictures, the maximum strain in two structural implant system is $0.37 \mathrm{~mm}$ and $0.027 \mathrm{~mm}$ respectively. In the implant, the areas of largest stress are the parts of largest strain, with the values of $0.00146 \mathrm{~mm}$ and $0.00143 \mathrm{~mm}$. With regard to the alveolar, maximum strain is located at the bottom of cavity in cancellous bone, with the values of $0.37 \mathrm{~mm}$ and $0.027 \mathrm{~mm}$.

In comparison with solid structural implant system, hollow structural implant system is subjected to larger stress and strain, the maximum stress of implant in hollow structure is $0.8 \%$ and the strain is $0.00003 \mathrm{~mm}$ more than solid structure .In conclusion, as for implant, the differences in strain and stress are too small to cause obvious influence in mechanical properties. The results of FEA show that the stress and strain are both keep in small values and don't reach the yield strength of samples based on SLM technique.

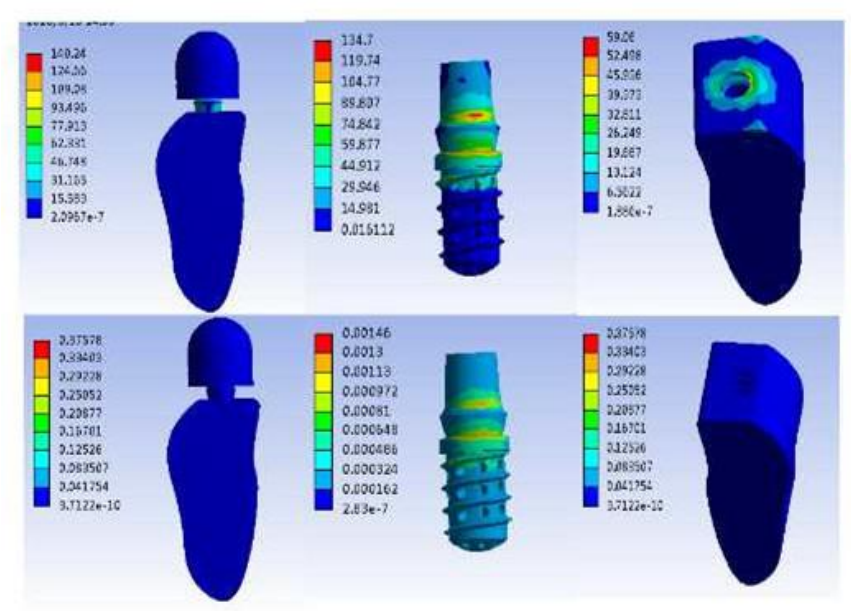

(a)

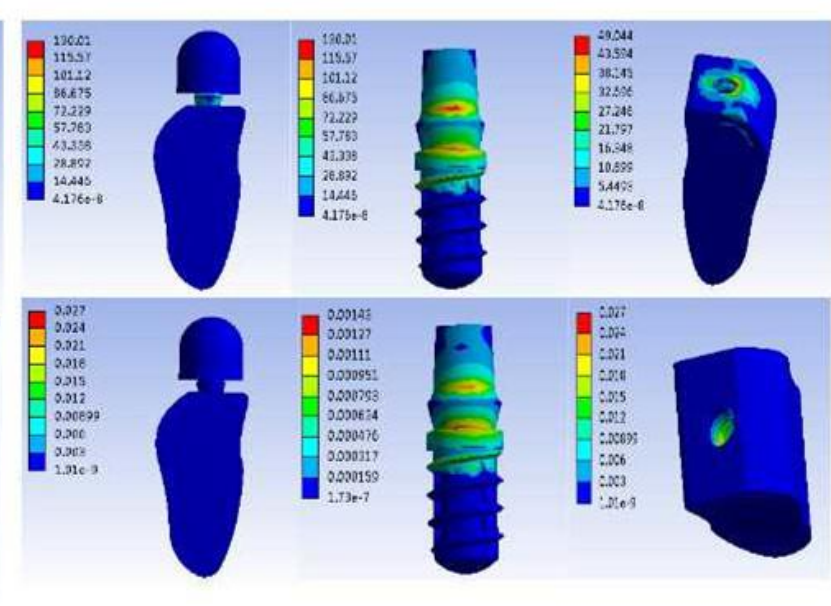

(b)

Fig. 2. The distribution of stress and strain (a), hollow implant system (b) solid implant system.

From the Fig. 3 (a), (b), the dental implant is still in a safe state after 5 million times cyclic loading and fatigue fracture phenomenon doesn't happen with the minimum safety factor of 7.0. The distribution of implant fatigue safety coefficient is in line with stress distribution basically. The areas with larger stress have lower level in safety factor such as the 
implant-abutment surface, abutment-crown surface and porous structure at the first lap of thread which are in higher stress, with the values between 7.0 and 13.0 in hollow implant and 7.9 and 13 in solid implant. It is believed that fatigue damage happens on the three areas with higher stress firstly.

a
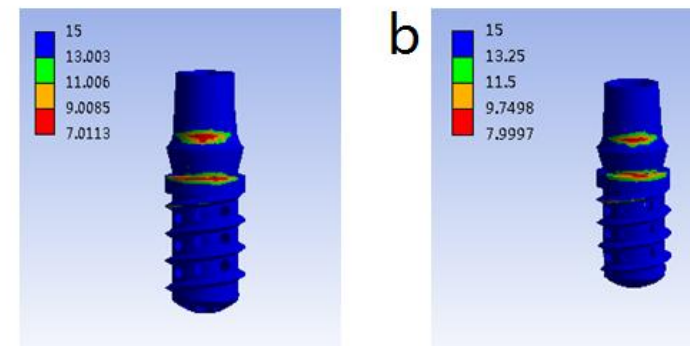

Fig. 3. Fatigue safety factor of dental implant. (a) hollow implant (b) solid implant.

\section{MANUFACTURE OF SAMPLES}

Pure titanium is widely used to manufacture dental implant in SLM technique due to the good biocompatibility and high corrosion and mechanical resistance. What is more, the elastic modulus is high relative to bone tissue. With this advantage, it can accelerate the ingrowth of tissue. In this study, TA2 is used for formed materials. SLM technique build parts layer by layer, from the bottom up, by scanning a focused laser or electron beam that melts a powdered raw material and promotes its consolidation. A powder is uniformly distributed over each layer prior to the subsequent layer scanning. The geometry and internal architecture of the parts are controlled by selectively applying the laser or electron beam power to the desired areas of consolidation.

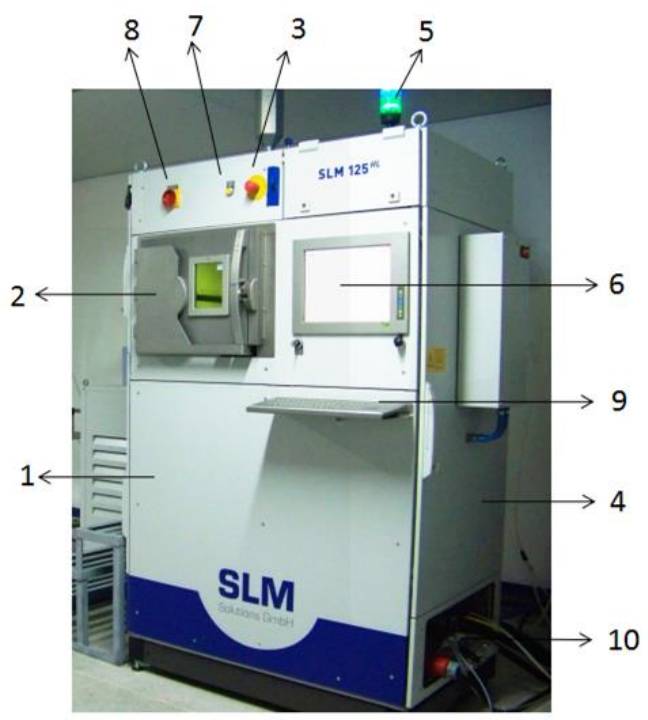

Fig. 4. The fabrication setup in SLM125HL YLR-100-WC; 1.process cabinet 2.process chamber 3.emergency STOP button 4.control cabinet 5.signal lamp 6.screen 7.[RESET] button 8.main switch with emergency STOP function 9.keyboard 10.connctions.

SLM125HL YLR-100-WC of SLM Solution company is used to study SLM technology in this paper, as is shown in Fig. 4. The size of machinable parts is $125 \mathrm{~mm} \times 125 \mathrm{~mm} \times 75 \mathrm{~mm}$, and the scanning speed is $15 \mathrm{~cm} 3 / \mathrm{h}$, layer thickness can be controlled from $20 \mu \mathrm{m}$ to $75 \mu \mathrm{m}$, the scanning spot size is $70 \mu \mathrm{m}$ to $130 \mu \mathrm{m}$, the minimum processing thickness is $140 \mu \mathrm{m}$ to $160 \mu \mathrm{m}$. The dimensional accuracy, porosity, level of oxygen and nitrogen and the mechanical properties for a given relative density were influenced by the energy input as journal paper [11]. Based on the experiment results, the optimum technical parameters are shown in Table II.

TABLE II: PROCESSING PARAMETER OF SLM TECHNIQUE

\begin{tabular}{llllll}
\hline \hline $\begin{array}{l}\text { process } \\
\text { parameters }\end{array}$ & $\begin{array}{l}\text { laser } \\
\text { powder }\end{array}$ & $\begin{array}{l}\text { scannin } \\
\text { interval }\end{array}$ & $\begin{array}{l}\text { scanning } \\
\text { speed }\end{array}$ & $\begin{array}{l}\text { layer } \\
\text { thicknes } \\
\text { s }\end{array}$ & $\begin{array}{l}\text { Scanning } \\
\text { spot }\end{array}$ \\
\hline value & $100 \mathrm{w}$ & $0.13 \mathrm{~mm}$ & $255 \mathrm{~mm} / \mathrm{s}$ & $70 \mu \mathrm{m}$ & $87 \mu \mathrm{m}$ \\
\hline \hline
\end{tabular}

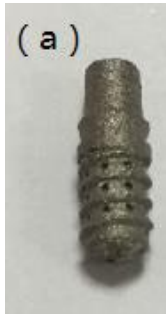

(b)

Fig. 5. The samples based on SLM (a), solid structure (b), hollow structure.

Two samples are manufactured according to the above parameters based on SLM technique, as shown in Fig. 4(a), (b). SLM technique can produce the whole parts without macroscopic defects. However, both the processing parameters resulted in small spherical particles visible on the surface. As for interior structure, some balling phenomenon is caused by temperature gradient and scanning interval. Therefore, the energy density of SLM machine has different degrees influence on the quality of samples with different materials, porosity and structure. It is the main direction for us to study the parameters of SLM technique in different structures.

\section{CONCLUSION}

In this study, relevant conclusions are listed in follows according to FEA and manufacture in porous structures:

1) Hollow implants basically satisfy the mechanical properties to bear occlusal force and save materials compared with solid structure based on SLM technique. All of the models are in safe states has been shown that proper hollow structure would not reduce the strength of the implant and result in loss of the fatigue fracture in this case. The stress concentration area may be broken according to fatigue and static analysis. However, some unreasonable hollow structure would lead to inadequate strength.

2) The samples based on SLM technique don't have obvious defects. But there is much balling phenomenon in their inner structure and around the pores. Therefore, appropriate structure should be chosen carefully in implant design. Effect of different designs based on SLM technique on the stress and strain distribution still need to be investigated. What is more, setting parameters according to different structure in SLM machine is the main problem to study new processes.

\section{ACKNOWLEDGMENT}

This work is supported by the Science and Technology Program of Shenzhen, China (No.JCYJ20140617143643469) 
and Foshan Innovative and Entrepreneurial Research Team Program, China (No.2014IT100102); Science and Technology Program of Shenzhen, China ( No.JCYJ20140617143643469; Shenzhen Science and Technology Innovation Committee (No.CXZZ20140414170821148); Foshan science and technology innovation special fund project (Foshan Cooperation Projects, No. 2014HT10008).

\section{REFERENCES}

[1] R. D. Li, "The key to the selective laser melting metal powder forming basic question research," Huazhong University of Science and Technology, 2010.

[2] J. Y. Chen, Z. G. Zhang, and Z. F. Li, "Selective laser melting technology application in the field of oral medicine," International Journal of Oral Medicine, vol. 1, pp. 97-101, 2014.

[3] L. Baggi, I. Cappelloni, M. D. Girolamo, F. Maceri, and G. Vairo, "The influence of implant diameter and length on stress distribution of osseointegrated implants related to crestal bone geometry: A three-dimensional finite element analysis," J. Pros. Dent., vol. 100, no. 6, pp. 422-431, 2008.

[4] O. Eraslan and Ö. İnan, "The effect of thread design on stress distribution in a solid screw implant: A 3D finite element analysis," Clinical Oral Investigations, p. 144, 2010.

[5] F. A. Fontijn-Tekamp and A. P. Slagter, "Bite forces with mandibular implant-retained overdentures," Dent Res., vol. 77, no. 10, pp. 1832-1839, 1998.

[6] E. Kitamura, "Biomechanical aspects of marginal bone resorption around osseointegrated implants: Considerations based on a three-dimensional finite element analysis," Clin Oral Impl Res., vol. 15, no. 4, pp. 401-412, 2004.

[7] K. Gotfredsen, T. Berglundh, and J. Lindhe, "Anchorage of titanium implants with different surface characteristics: An experimental study in rabbitslnt J Clin Implant," Dent Relat Res, vol. 2, no. 3, pp. 120-128, 2000.

[8] M. Esposito, "Biological factors contributing to failures of osseointegrated oralimplants," Etiopathogenesis, vol. 106, no. 3, pp. 721-764, 1998.

[9] X. W. Ren, L. Zeng, Z. M. Wei, X. Z. Xin, and B. Wei, "The effects of multiple firings on metal-ceramic bond strength of $\mathrm{Co}-\mathrm{Cr}$ alloy fabricated by selective laser melting".

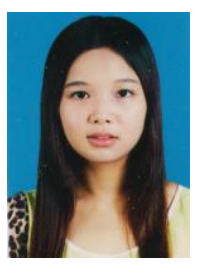

Yaling Wang was born in Sichuan, China, in 1992. She started her bachelor study in School of Manufacturing Science and Engineering at Southwest University of Science and Technology. Then she continued her master degree in Chongqing University in 2014.

She is an assistant Engineer of Foshan Angels Biotechnology Co., Ltd. Her major research field is mechanical engineering, especially in mechanical design.

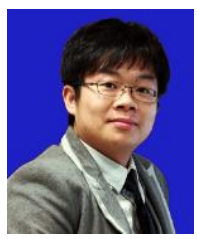

Xianshuai Chen was born in Guangxi, China, in 1984. He started his bachelor study in Harbin Institute of Technology. Then he continued his Ph.D. in Chinese University of Hong Kong in 2009.

$\mathrm{He}$ is a managing editor of "Chinese Journal of Tissue Engineering Research", member of Youth Innovation Promotion Association CAS, Returned overseas talents, associate professor of Guangzhou institute of advanced technology, Chinese academy of sciences and Chinese University of Hong Kong. His major research field is mechanical engineering, especially in precision engineering.

Prof. Chen is executive director of Guangzhou Janus Biotechnology Co. Ltd. He has published over 20 articles in foreign journals and conference, of which 20 have been indexed in the SCI.



Yuanxin Luo was born in Guangxi, China, in 1983. He obtained his bachelor and master degree in Chongqing University. Then he continued his Ph.D. in Chinese University of Hong Kong in 2008.

$\mathrm{He}$ is associate professor and assistant to the dean in Chongqing University. His major research fields are plastic forming technology and Material - fatigue creep. Prof. Luo has hosted Youth fund of national natural science fund, State key laboratory open fund, the natural science foundation of Chongqing Municipal Science and technology commission andfour enterprise horizontal topic as project leader. He has taken part in two mega project of science research as leading researcher. He has published over 20 articles in foreign journals and conference, of which 10 have been indexed in the SCI.

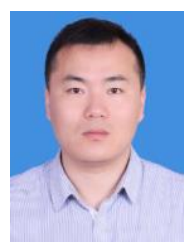

Chunyu Zhang was born in Heilongjiang, China, in 1985. He has obtained his bachelor and master degree.

$\mathrm{He}$ is a senior engineer of Guangzhou Janus Biotechnology Co., Ltd. His major research field is materialbprocessing, especially in military products and the design plastic forming of aerospace products.

Mr. Zhang has participated in a series of national key scientific research projects. Now he is working on the fields of SLM technique and has published more than 7 articles in all kinds of journals and academic conference and 35 patents.

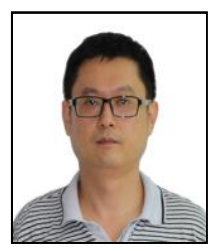

Peng Zhang obtained his bachelor degree in Jiamusi University. His major research fields are Maxillary sinus lift in ascension, bone extrusion bone splitting technique and immediate implanting and repair technology.

$\mathrm{He}$ is an oral physician of Foshan Stomatology Hospital, director of Foshan stomatological association, vice director of tooth pulp committee, member of oral-repair association.

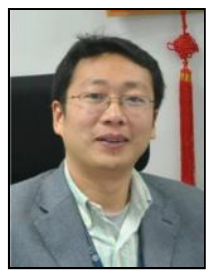

Wei Feng was born in Hubei, China in 1980. He received the B.S. degree in School of Materials Science and Engineering, Huazhong University of Science and Technology (HUST), China, in 2001. He received the $\mathrm{Ph}$. D. degree from HUST in 2006.

$\mathrm{He}$ is now an associate professor of Shenzhen Institute of Advanced Integration Technology, Chinese Academy of Sciences and the Chinese University of Hong Kong. His research interests include robotics and intelligent system, computational geometry and computer graphics, $\mathrm{CAD} / \mathrm{CAM} / \mathrm{CAE}$ 